\title{
Don Ricardo Palma, hoy
}

\author{
Alberto Varillas Montenegro \\ Academia Peruana de la Lengua \\ Instituto Riva Agüero \\ varillas.alberto@gmail.com \\ Lima-Perú
}

\section{Resumen}

Es muy corriente que se presente a Ricardo Palma como un escritor de avanzada edad que escribió las Tradiciones Peruanas. Este trabajo está destinado a recordar que hoy se dispone de toda la información necesaria sobre el origen, nacimiento, estudios escolares y movida juventud de Palma. En la actualidad, se conoce el detalle de su paso por la Marina peruana, sus andanzas como periodista liberal y sus dos destierros, su larga vida como correcto funcionario público; se sabe, también, con el éxito de sus más de 500 tradiciones, así no hubiera creado un género nuevo y haber rescatado de la nada a la Biblioteca Nacional, que se constituyó en uno de los más valiosos promotores de la reacción peruana luego de la Guerra del Pacífico.

Palabras clave: Palma biografía, triunfo político, tradicionista, Bibliotecario mendigo

\section{Abstract:}

It is very common for Ricardo Palma to be presented as an elderly writer who wrote the Peruvian Traditions. This work is destined to remember that today we have all the necessary information about the origin, birth, school studies and moving youth of Palma. At present, we know the detail of his time in the Peruvian Navy, his adventures as a liberal journalist and his two exiles, his long life as a correct public official. We also know that with the success of his more than 500 traditions, even if he would not have created a new genre or would not have rescued from nothing the National Library, he became one of the most valuable promoters of the Peruvian reaction after the War of the Pacific.

Keywords: Biography of Palma, political triumph, traditionist, Beggar Librarian 
Alberto Varillas Montenegro (Perú): Miembro de número de la Academia Peruana de la Lengua (Vicepresidente), la Academia Nacional de la Historia, el Instituto Riva Agüero y el Instituto Ricardo Palma. Se ha especializado en la historia y la literatura del Perú del siglo xix. Entre otras obras ha publicado Felipe Pardo y Aliaga, La literatura del Perú del siglo xix, Perú y Ecuador: Visión actual de un antiguo conflicto, Periodismo e Historia del Perú en el siglo xix, Apuntes para una historiografía de la literatura peruana del siglo XIX. Ha editado las Obras completas de Manuel A. Segura. 
A fines del siglo pasado, una importante conocedora de nuestra literatura observaba que en las tres décadas anteriores la crítica había centrado su interés en la vida de Ricardo Palma pero que no mostraba mayor preocupación por la más importante de sus obras, es decir, por las Tradiciones peruanas. Hoy, veinte años después, es posible complementar aquella observación añadiendo algunas precisiones: la imagen personal del ilustre tradicionista que viene interesando a la crítica y que se trasmite a sus lectores es más la del "viejecito zumbón" como lo aludió Luis Fernán Cisneros hace casi un siglo, o el retrato idealizado a tinta con que lo captó Jorge Holguín de Lavalle o la del anciano del famoso oleo de Teófilo Castillo, obras de arte que se encuentran hoy en la Casa Museo Ricardo Palma.

A cien años del fallecimiento del tradicionista, pretendo apartarme de la tónica académica que tienen los trabajos que se presentan anualmente en la importante fiesta palmista que anualmente convoca el Instituto Ricardo Palma en el mes de octubre, la Re-visión de las Tradiciones, y trazar un perfil equilibrado de uno de los más importantes exponentes de la cultura del Perú republicano y limpiar la imagen de Palma de todas las medias verdades que sobre él se han trazado a lo largo de más de cien años.

\section{El personaje}

En primer lugar, repitamos que Palma fue limeño y que nació en una de las zonas más antiguas de la capital peruana, al costado del edificio del Tribunal de la temible Inquisición y a menos de cinco cuadras de la Plaza mayor, y que se le bautiza como Manuel Palma en la parroquia del Sagrario el 11 de febrero de 1833. Por lo tanto, conviene descartar definitivamente las diferentes propuestas que se han formulado sobre la fecha y lugar de su nacimiento que a partir de 1915 formularon, entre 
otros, el venezolano Rufino Blanco Fombona mal informado por González Prada, el futuro director de la Biblioteca, Carlos A. Romero y Luis Humberto Delgado, propuestas que tan bien ha estudiado y descartado Oswaldo Holguín (1994).

Manuel Palma nace como hijo de Pedro Palma, comerciante minorista -se le podría considerar mercachifle, es casi seguro que ambulante- natural de Cajabamba (Cajamarca) y de Dominga Soriano, joven de 16 años, originaria de Cañete, tierra de explotación agrícola en cierto modo cercana a Lima, caracterizada por la cantidad de esclavos que la cultivaban. Como por razones de edad Dominga Soriano no podía contraer matrimonio, la madre de esta, Guillerma Carrillo, la declara como propia, situación que se corrige cuando Dominga llega a la mayoría de edad. Equivocándose, el bautizante consignó en la partida que ambos padres eran pardos, cuando consta que el padre, 15 años mayor, era mestizo. La partida de Bautismo de Ricardo Palma sirve para ubicar socialmente a sus padres: el padrino y los testigos aparecen como "don" fulano de tal: los padres de la criatura, no. Pese a la evidente discriminación, la economía de Pedro Palma no fue mala y, si bien fue un mercachifle o buhonero, gozó de un relativo éxito y llegó a tener cierta prestancia dentro de su gremio.

Los Palma -en especial don Pedro- cuidaron bien a su hijo, por entonces Manuel, y en todo momento fueron retribuidos por él con sus destacadas notas escolares. Como en aquella época ocasionalmente se publicaban en algunos diarios limeños las notas de los buenos alumnos, hoy reparamos en que el tradicionista había destacado en francés, matemáticas, contabilidad y nociones de economía política.

Durante sus últimos años escolares -cuando era alumno en el colegio de Andrés Orengo en 1846- o quizás en los primeros en que estuvo vinculado al Convictorio de San Carlos, el 
aún Manuel Ricardo Palma recorría la ciudad capital que seguramente conoció de palmo a palmo. De la misma manera, este inquieto quinceañero cruzaba las murallas que cercaban Lima desde la época del virrey Melchor de Navarra y Rocafull, Duque de la Palata (1685), y se internaba en algunas de las zonas aledañas como la Alameda de los Descalzos y la Carretera de Amancay hoy conocida como la Alameda de los Bobos, o las cercanías al estanque de Santa Beatriz y el callejón de Matamandinga, por el otro extremo de la ciudad. Y estos años dejaron recuerdo en el futuro escritor pues él mismo las recuerda como "Bienaventuradas horas, en las que nos imaginábamos orégano todo el monte" (2014, p. 73).

Por aquellos años, también, el joven Palma -como todos los jóvenes a esa edad- quería dejar de depender de su familia para lo cual necesitaba agenciarse recursos y él mismo cuenta que preparaba algunas composiciones que una monja clarisa relacionada con su familia publicaba como suyas y le retribuía con exquisitos dulces y pastillas de briscado. ${ }^{1}$ Cuando tenía entre quince y dieciséis años, encontró otra forma de llenarse el bolsillo: en la librería de Pérez en la calle de las Mantas, hoy primera cuadra del jirón Callao, había una sección de alquiler y canje de libros: el futuro e ingenioso escritor visitaba el establecimiento con el pretexto de "cambiar las obras que algunos de sus compañeros [del Convictorio] le encomendaban" (Palma, 1948, p. 86) y por cierto algo le quedaba por su gestión. Pero la misma librería de Pérez, centro de reunión de políticos y escritores, le ofreció otra oportunidad de obtener algún dinerillo y se entiende que desde muy joven puede haber colaborado en alguna de las publicaciones periódicas cuyos editores recalaban en la librería $\mathrm{y}$ es posible que haya preparado algunas notas -rentadas, por supuesto- para la crónica teatral o textos breves para otras columnas de limitada importancia. Pero aún otra forma de

l Es decir, dulces con mucha decoración. 
obtener recursos llegó a tener Palma: transcribir documentos que requerían los investigadores que pasaban por la Biblioteca Nacional y de allí nace, por ejemplo, su amistad con Juan María Gutiérrez, el importante estadista, jurisconsulto y escritor argentino.

No es posible olvidar que fue por aquellos años en que el futuro tradicionista toma contacto con la Marina peruana. El 7 de febrero de 1852, Palma, cenando con don Miguel del Carpio, importante político y Mecenas de los románticos peruanos, junto a su cubierto encuentra un sobre que contenía su nombramiento como oficial del Cuerpo Político de la Armada, lo que hoy se llamaría empleado administrativo de la Marina. En setiembre del año siguiente, ante la fuerte presión de una posible futura suegra indignada, el joven poeta consulta con del Carpio sobre un posible y precario matrimonio y recibe como respuesta "iCasarse a los veinte años! iQué disparate!" (Angélica Palma, 1933: 23) y de inmediato logra embarcarlo en la goleta Libertad, en la condición de Contador, con lo que da inicio a su vinculación marítima con la Marina peruana.

\section{Palma, correcto funcionario público}

Lo anterior nos da pie para poner de relieve la condición de correcto funcionario público que observó Palma durante toda su vida, lo que aquí y ahora resulta sumamente importante destacar. En tres momentos de su larga vida presta servicios al Estado don Ricardo Palma: a partir de 1852, dentro del ministerio de Guerra y Marina, desde 1868 hasta 1873, como secretario personal del presidente Balta y senador por Loreto, y entre 1883 y 1912 como director de la Biblioteca.

La enumeración anterior podría ser tachada de parcializada si no se recuerdan algunas interrupciones en su quehacer 
administrativo: su adhesión al intento revolucionario de 18561857 contra el presidente Castilla, su participación en el levantamiento que pretendió capturar el domicilio del general Castilla en 1860 y su consecuente expatriación del país durante dos años, y su frustrado intento de asumir una función consular en Belém, Brasil, entre 1863 y 1864, lo que le valió conocer algunos países de Europa, Brasil, Cuba y Estaos Unidos. Cada una de estas andanzas estuvo rodeada de razones distintas y si bien puede considerarse que no deben extrañar totalmente dado el nivel de desorden del Perú y la mayoría de sus instituciones durante aquellos años, deben ser censuradas puesto que no se limitaron a ser únicamente mataperradas.

La vinculación de Palma con la Armada peruana fue estrecha. Se inició en la goleta Libertad y continuó en diferentes embarcaciones y en tierra, en el Cuerpo político. El punto culminante de su actuación como embarcado fue su destacada actuación a raíz del hundimiento del Rímac y en los días que siguieron cuando fue necesario tratar de salvar a los náufragos que habían llegado a las desiertas playas de Acarí. Lo cual le valió una felicitación especial.

Nuestro escritor se incorporó al grupo liberal levantado en armas contra el gobierno del general Prada que, en respaldo de su posición, traía el mérito de haber sido él quien había derrotado al pusilánime presidente Pezet y triunfado en la guerra contra España. En varias tradiciones recuerda Palma sus andanzas con el coronel Balta en el norte peruano hasta el triunfo revolucionario y su incorporación como secretario del ya electo presidente Balta y su elección como senador por el departamento de Loreto. Lamentablemente, las memorias en que recuerda los detalles de su participación durante la administración Balta se perdieron cuando su domicilio miraflorino fue incendiado durante la guerra del Pacífico y se quemó el manuscrito que había preparado. Pero los Diarios de 
debates registran sus intervenciones en el Congreso nacional hasta mediados de 1873.

Pero merece párrafo aparte destacar la importante participación de Palma dentro del ámbito de la Biblioteca hoy Nacional. Desde muy joven, Palma merodeaba alrededor de la Biblioteca buscando libros, folletos y manuscritos. La seriedad de sus actividades dentro del importante establecimiento que había fundado el general San Martín debe haber sido notoria puesto que su director, el benemérito Gonzáles Vigil, lo recomendaba cada vez que algún investigador requería búsquedas o transcripciones. En 1880, el dictador Piérola nombra a Palma subdirector ad honorem de la Biblioteca, seguramente con el propósito de que apoye a su director, el anciano general Odriozola, y en esa condición lucha al año siguiente para evitar que las fuerzas invasoras culminaran con éxito la depredación y prepara la carta de protesta que, suscrita por el director, se depositó en las representaciones diplomáticas acreditadas en el Perú invadido. Suscrito el Tratado de paz de Ancón y liberada la capital de las tropas saqueadoras, Palma acepta del gobierno el cargo de Director de la Biblioteca y Archivo Nacional, desdeñando la jugosa oferta económica que le había formulado desde Buenos Aires el diario La Prensa. Desde noviembre de 1883 hasta febrero de 1912 -casi treinta años- Palma desempeña el cargo y a base de su prestigio personal y su encomiable esfuerzo logra reabrir la Biblioteca en julio de 1884 con un número de volúmenes casi igual al que había conservado su estantería hasta antes de la guerra. En poco tiempo había convertido un local que había quedado destrozado pues había servido de cuartel y de caballerizas, que carecía de los materiales bibliográficos mínimos, que no tenía estantería ni personal ni recursos con que agenciárselos en una institución nueva muy útil para el desarrollo de la cultura peruana. Por esos méritos y por haber sido la Biblioteca la primera muestra del renacimiento del Perú 
que siguió a la tragedia, en medio de las dificultades internas y la guerra civil que se veía venir, la figura de Palma resulta constituyéndose como una de las primeras y más importantes de la recuperación nacional.

Fue Palma, pues, un eficiente y correcto funcionario público que mostró su competencia y honestidad cada vez que tuvo oportunidad.

\section{Palma escritor}

Dentro de la cultura peruana republicana, la figura de Palma tiene un sitial de inmensa importancia. Fue un buen exponente de la literatura romántica peruana, un historiador confiable, un entusiasta periodista, un lexicógrafo destacado. Pero lo fundamental de su producción es haber creado un género literario que, como lo ha demostrado Estuardo Núñez, se extendió por toda Hispanoamérica (1998).

\section{Palma poeta romántico}

En el tardío romanticismo peruano, Palma fue uno de los más caracterizados de sus representantes. Como todos los integrantes de aquel movimiento, Palma comenzó con el teatro y la poesía, pero sus aportes en ambos géneros fueron víctimas de su propia autocrítica.

La producción en verso del tradicionista se encuentra en seis volúmenes publicados a lo largo de más de veinte años: Poesías (Lima, 1855), Armonías (París, 1865), Lira Americana (París, 1865), Semblanzas (Lima, 1867), Pasionarias (Le Havre, 1867) y Verbos y gerundios (Lima, 1877). Posteriormente publicó Filigranas (1892) y preparó varias ediciones de poesías completas que en realidad son recopilaciones seleccionadas y corregidas 
en las cuales no fue especialmente generoso al recoger sus composiciones juveniles. Al final de su vida, Palma reconocerá que “...todo el cariño literario que abrigo por mis Tradiciones o leyendas en prosa solo pueden igualarse al desapego que siento por mis renglones rimados" aunque casi de inmediato reconocerá que “... con versos me inicié en la labor literaria, y pecaría de ingrato si me creyera con derecho para renegar de ellos" (Palma, 191 1, p. 5)

Recientemente se ha vuelto a publicar un artículo de un tercio de siglo de antigüedad, en que un conocido palmista, Luis Jaime Cisneros, enfatiza que "Palma también escribió versos" y recordando a Luis Benjamín Cisneros, prologuista de uno de los poemarios de Palma, advierte de que

Si Juvenilia representaba todavía la inexperiencia de Palma en los años primeros, y si en Armonías se insinuaba ya la laboriosa e ilustrada juventud, ve en Pasionarias al poeta maduro y anuncia que "el arte de la forma" ha alcanzado consagración $(2019$, p. 83).

Lo cierto es que hasta la fecha la obra poética de Palma no ha merecido el estudio serio que, dada la importancia del autor, merece salvo los comentarios ya algo antiguos de Washington Delgado (1980) al historiar la literatura republicana nuestra.

\section{Palma autor teatral}

El movimiento romántico tuvo una adicción al teatro. Debe recordarse que fue con el drama Hernani de Víctor Hugo y la batahola que se produjo luego de su estreno (1830) con que se reconoce la aparición pública del romanticismo francés, y que con el Don Álvaro o la fuerza del sino, del Duque de Rivas (1835) ocurre lo mismo en España. Y siempre se ha dicho 
que ya en la década de 1850 los jóvenes románticos peruanos fracasaron cuando quisieron hacer lo propio. Los románticos peruanos cultivaron el teatro con enorme entusiasmo pero sin mayor éxito aunque desde mediados de la década de 1840 sus primeras obras ya aparecían -e incluso se repetían- en los escenarios limeños.

Palma no fue ajeno a esta vocación y escribió dramas y comedias y tradujo obras para el teatro local. Fue autor de cuatro dramas con tema patriótico: El hijo del sol (1849), La muerte o la libertad, La hermana del verdugo y Rodil (1851). Pocos años después optó por las petipiezas, obras cómicas compuestas en un acto, muy difundidas en el siglo xix, y tres muestras de su ingenio llegan a las tablas limeñas en la misma década: Los piquines de la niña (1855), Criollos y afrancesados (1857) y iSanguijuela! (1858).

Palma no se mostró satisfecho de su teatro y destruyó los textos de todas sus obras en este género aunque, contra su voluntad, se salvó un ejemplar de Rodil que José Jiménez Borja descubrió en 1952 y que no hizo sino confirmar la opinión del autor en el sentido de que su teatro no era especialmente meritorio.

\section{Palma novelista}

Refiere Palma que había escrito una novela, Los Marañones, historia de Lope de Aguirre y sus aventuras en la selva peruana en 1541-1542. Lamentablemente, el manuscrito de la obra de la cual nada se conoce- se quemó durante el incendio del domicilio miraflorino de Palma, durante la Guerra con Chile, el 15 de enero de 1881. Dados los rasgos del personaje, hubiera sido interesante conocer la visión que sobre él tenía el tradicionista. 


\section{Palma periodista}

Como todos los escritores jóvenes de mediados de la antepasada centuria, Ricardo Palma se sintió muy pronto atraído por el periodismo. Si bien hay testimonio de lo dilatada de su vocación periodística -desde una poesía en El Comercio de Lima en 1848 hasta una tradición en La Prensa de Buenos Aires en 1915, 167 años de interés por el periodismo!- seguramente fueron muchos más de los que hoy se conocen sus aportes al periodismo como cronista y redactor, como versificador satírico, autor de Tradiciones, etc. En los últimos años se han recopilado los despachos que remitía a Panamá para El Canal, periódico favorable a la causa peruana en la Guerra del Pacífico, de donde se han rescatado y aparecen publicadas muchas de sus Tradiciones, inclusive hace pocos años se ha recuperado aunque en muy mal estado- el semanario El Burro, del cual se ignora si fue dueño o copropietario, pero que le mereció un comentario manuscrito suyo que aparece en la única colección que se conoce y que se encuentra en la Hemeroteca de la Biblioteca Nacional: "Fue este el primer periódico que redacté en mis años de colegial. Despapuchos [ilegible] de un muchacho de 19 años. De buena gana quemaría hoy estas burradas. Lima, 1885. R.P."

El periodismo, como el propio Palma lo declara, le permitió cobrar su primer salario pero, salvo el caso de La Prensa de Buenos Aires que por patriotismo desdeñó, nunca le ofreció la oportunidad de dedicarse a él en forma permanente.

\section{Palma lexicógrafo}

Palma tuvo un marcado interés por el idioma que hablaban los americanos y, en concreto, los peruanos. Con Pedro Paz Soldán y Unánue (Juan de Arona) fueron los dos lexicógrafos peruanos 
de mayor importancia del siglo XIX. La vocación de Palma por el idioma fue muy antigua y se acredita con su interés por remitir a la Real Academia Española papeletas con términos que iban siendo admitidos como americanismos o peruanismos. Precisamente el interés de Palma en lograr la aceptación de más de quinientas voces y la negativa de la Academia en aceptar la totalidad de las propuestas es lo que determina el pleito entre ambas Academias, la Española y la Peruana, en Madrid, y el receso entre 1893 y 1917 de la Academia correspondiente nuestra. ${ }^{2}$

De vuelta al Perú, Palma publicará dos obras en el campo de la lexicografía: Neologismos y Americanismos (1896) y Papeletas lexicográficas (1903) y en esta última declarará que ya estaba despojado "de [la] gran irritabilidad nerviosa" (Palma, 1903, p. v) con que había escrito la primera de ellas y continúa desarrollando su labor lexicográfica.

\section{Palma historiador}

La labor de Palma como historiador se aprecia en dos textos conocidos: su Anales de la Inquisición de Lima (1863) y su Monteagudo y Sánchez Carrión. Páginas de la historia de la Independencia (1877), meritorio trabajo el primero y tremendamente polémico el segundo, pues generó un debate internacional en el que intervinieron los partidarios de Bolívar de diversos países de América. Como ya se ha indicado, Palma redactó también un trabajo titulado Reminiscencias de la administración del coronel Balta, dedicado a recordar los años del gobierno de Balta que conoció tan bien (1878-1872), cuyos originales fueron víctimas del incendio de su domicilio, después de la batalla de Miraflores, durante la Guerra del Pacífico.

2 Buen número de los americanismos propuestos por Palma fueron aceptados en esa oportunidad por la Academia madrileña; sin embargo, otros no lo fueron lo que motivó la indignación del tradicionista y la recesión de la Academia peruana. 
Quizás sea necesario aquí incorporar una breve aclaración sobre las Tradiciones peruanas como historia. Palma nunca tuvo interés en conceder a sus tradiciones el carácter de fuente histórica. Su lectura, si bien incluye datos y fechas susceptibles de ser analizadas con mayor detenimiento, proporciona al lector, sin embargo, una idea bastante acertada de la forma de vida durante la cual se desarrollan. Fue el dominio de Palma en este campo, lo que el serio y riguroso Manuel González Prada no logró captar. Al final de su vida, Palma precisó su posición: "La tradición, a lo sumo, es un auxiliar de la historia porque despierta en el lector la curiosidad por investigar un hecho y consultar o beber agua en mejor fuente" (1907, p. 37).

Y al prologar a Clorinda Matto de Turner, entusiasta seguidora de las Tradiciones, es más amplio en su descripción:

La Tradición no es más que una de las formas que puede revestir la historia, pero sin los escollos de esta. Cumple a la historia narrar los sucesos secamente, sin recurrir a las galas de la fantasía, y apreciarlos desde el punto de vista filosófico o social. A [la tradición] sobre una pequeña base de verdad, le es lícito edificar un castillo. El tradicionista tiene que ser poeta y soñador. [...] Estilo ligero, frase redondeada, sobriedad en las descripciones, rapidez en el relato, presentación de personajes y caracteres en un rasgo de pluma, diálogo sencillo a la par que animado, novela en miniatura, novela homeopática, por decirlo así, eso es lo que, en mi concepto, ha de ser la Tradición (Palma, 1999, p.73).

\section{Palma tradicionista}

El gran mérito de Palma radica en que inventó un nuevo género literario. Isabelle Tauzin (1999), después de un detenido análisis 
de las tradiciones y de las opiniones de Palma sobre este nuevo género literario, atribuye tres características a la tradición: (1) su heterogeneidad, es decir, está conformada por la mezcla de rasgos de distintos géneros: no se limita al ineludible vínculo con la historia del Perú pero añadiéndole la comicidad de una situación, el drama de una vida o la ingeniosidad de un chiste; si bien tiene raíces costumbristas, no es básicamente descriptiva; aunque tiende a acumular anécdotas, alterna relatos de distinta extensión con coplas y refranes, episodios graciosos. Esta heterogeneidad sin límites, insiste Tauzin, constituye al fin y al cabo la verdadera originalidad de la tradición; (2) la brevedad, que es lo que condujo a Manuel González Prada a considerarla con algo de ligereza como una 'caricatura microscópica de la novela'; y (3) el ingenio, que fue el verdadero aporte de Palma pero que ninguno de sus múltiples seguidores supo utilizar (1999, p. 225-226).

Y Tauzin escoge acertadamente una frase de Palma quien describe a su amigo el académico español Vicente Barrantes cómo en la tradición se unen forma y fondo:

Una tradición no es un trabajo ligero, sino una obra de arte. Tengo paciencia de benedictino para limar y pulir la frase. Es la forma más que el fondo, lo que las ha hecho tan populares (p. 226).

Y al guardar la pluma después de haber redactado más de 500 tradiciones, don Ricardo Palma se vio encumbrado a los niveles más altos de la literatura hispanoamericana y consagrado como el primer escritor republicano del Perú.

\section{Palma en su contexto}

Como se ha visto, don Ricardo Palma fue un hombre múltiple, con aceptable éxito entre los integrantes de la tardía generación 
romántica peruana dentro de la cual fue un más que aceptable versificador, entusiasta hombre de teatro, controvertido historiador, entretenido periodista y perseverante lexicógrafo. Sin embargo, al haber logrado dar forma al género que había estado desarrollando desde sus años mozos, la tradición, y comenzado a publicar sus Tradiciones peruanas, lentamente se desvincula del retrasado romanticismo peruano que, de otro lado, fue uno de los últimos en aparecer en Hispanoamérica y que surge cuando había sido totalmente superado en Europa, concretamente en España, y se convierte en el más importante escritor peruano republicano con una especial capacidad para influir en la literatura de buena parte de los países hispanoamericanos. La arrolladora personalidad que tuvo, la vasta cultura de la cual disfrutó y el ingenio innato con el que nació le permitieron a Palma abrirse campo dentro de un medio tan difícil como es la literatura nuestra y extender su influencia más allá de nuestros límites.

Pero al haber desdeñado una oferta generosa del diario La Prensa de Buenos Aires para asumir, en desmedro de la economía suya y de la de su propia familia, la Dirección de la Biblioteca y el Archivo Nacional en momentos en que esta carecía de un local medianamente decoroso, del material bibliográfico imprescindible, del personal y de los recursos para cubrir sus necesidades se convirtió no solo en el Bibliotecario mendigo, tal como el mismo se autodefinió, sino en uno de los paladines de la reacción nacional. En los aciagos años de la post guerra, a los que don Jorge Basadre ha mencionado como 'el Perú yacente', mientras las huestes de los generales Iglesias y Cáceres y políticos de todo pelaje disputaban -sin duda imbuidos de un indiscutible patriotismo- el poder en busca de una recuperación, fue Palma, valiéndose solo de su prestigio personal, el único que caminó hacia adelante y el 28 de julio de 1884 logró reabrir la Biblioteca poniendo a disposición de la 
ciudadanía tantas unidades bibliográficas como había habido antes de la conflagración. Prueba de lo eficaz de su gestión fue haber logrado que el presidente chileno Domingo Santa María devolviera 14.000 obras tomadas de nuestra Biblioteca por el ejército invasor como botín de guerra.

Es necesario, pues, destacar la labor de un Palma patriota a costa suya y contraído a su trabajo como muy pocos en ese momento, que desdeñó la diatriba para poner de manifiesto, como en el actual momento tantos debían hacer, que entre todas las alternativas la más importante era luchar por la salvación de la patria, el Perú.

\section{Bibliografía}

Cisneros, L. J. (2019). Sobre Ricardo Palma. pp. [73]-83. Lima: Editorial Universitaria de la Universidad Ricardo Palma.

Delgado, W. (1980). Historia de la literatura republicana. Lima: Ediciones Rikchay Perú.

Núñez, E. (1998). Ricardo Palma Escritor Continental. Lima: Fondo Editorial del Banco Central de Reserva del Perú.

Holguín Callo, O. (1994). Tiempos de infancia y bohemia. Ricardo Palma (1833-1860). Lima: Fondo Editorial de la Pontificia Universidad Católica del Perú.

Palma, A. (1933). Ricardo Palma. Buenos Aires: Ediciones argentinas "Cóndor".

Palma, R. (1903). Papeletas lexicográficas. Lima: Imprenta La Industria.

Palma, R. (1911). Poesías completas. Barcelona: Casa Editorial Maucci. 
Palma, R. (2014-2015). Tradiciones peruanas. Lima: Editorial Universitaria de la Universidad Ricardo Palma.

Palma, R. (2005-2006). Epistolario. Lima: Editorial Universitaria de la Universidad Ricardo Palma.

Tauzin Castellanos, I. (1999). Las Tradiciones peruanas de Ricardo Palma. Claves de una coherencia. Lima: Editorial Universitaria de la Universidad Ricardo Palma.

Recibido el 9 de octubre de 2019 Aceptado el 20 de octubre de 2019 